\title{
Centro de material e esterilização: acidentes de trabalho e riscos ocupacionais
}

\author{
Supply and sterilization center: work accidents and occupational hazards \\ Centro de material y esterilización: accidentes y riesgos laborales
}

Jael Maria de Aquino ${ }^{1}$, Lívia Pereira Barros² ${ }^{2}$ Sâmara Aline Brito ${ }^{3}$, Emanuela Batista Ferreira ${ }^{4}$, Sílvia Elizabeth Gomes de Medeiros ${ }^{5}$, Elizabeth Rafaela dos Santos ${ }^{6}$

RESUMO: Objetivo: Investigar a ocorrência de acidentes de trabalho e os principais riscos ocupacionais a que estão expostos os profissionais de Enfermagem em Centro de Material e Esterilização. Método: Estudo exploratório, transversal, de abordagem quantitativa, realizado em dois hospitais, com 37 profissionais de Enfermagem. Os dados foram coletados mediante questionário semiestruturado produzido pelas autoras, de agosto a setembro de 2012. Resultados: Verificou-se que 27,3\% dos profissionais da instituição pública já sofreram acidentes; destes, $83,3 \%$ do tipo perfurocortante. Na instituição privada, o percentual foi de $46,7 \%$, sendo $57,1 \%$ por queimaduras na autoclave. Conclusão: Dentre os acidentes de trabalho levantados, as lesões com perfurocortantes e as queimaduras por autoclave foram as mais representativas nas instituições. Ressalta-se a necessidade de atitudes que valorizem a prática do registro dos acidentes laborais, visando à implantação de medidas de prevenção, proteção e recuperação da saúde dos profissionais envolvidos na assistência direta e indireta à saúde.

PALAVRAS-CHAVE: Acidentes de trabalho; Riscos ocupacionais; Enfermagem.

ABSTRACT: Objective: To investigate the occurrence of occupational accidents and the main occupational risks that nursing professionals are exposed to in a Supply and Sterilization Center. Method: An exploratory, cross-sectional study of quantitative approach carried out in two hospitals with 37 nursing staff members. Data were collected through semi-structured questionnaires produced by the authors, from August to September 2012. Results: We verified that $27.3 \%$ of the professionals from the public institution suffered accidents; $83.3 \%$ of them were needlestick injuries. In the private institution, the percentage of accidents was $46.7 \%$, with $57.1 \%$ of them caused by burns in the autoclave. Conclusion: Among the workplace accidents surveyed, needlestick injuries and burns were the most representative in both institutions. We emphasize the need for attitudes that enhance the practice of recording occupational accidents, aiming at the implementation of measures of prevention, protection and health recovery of professionals involved directly and indirectly in health care.

KEYWORDS: Accidents, occupational; Occupational risks; Nursing.

RESUMEN: Objetivo: Investigar la ocurrencia de accidentes laborales y los principales riesgos laborales a que están expuestos los profesionales de enfermería en el Centro de Suministros y Esterilización. Método: Estudio exploratorio, transversal, de enfoque cuantitativo, realizado en dos hospitales, con 37 profesionales de enfermería. Los datos fueron recolectados a través de un cuestionario semiestructurado producido por las autoras, entre agosto y septiembre de 2012. Resultados: Se verificó que el 27,3\% de los profesionales de la institución pública sufrió accidentes, de los cuales el 83,3\% fue con objetos cortopunzantes. En la institución privada el porcentaje fue del 46,7\%, de los cuales el 57,1\% fue de quemaduras por autoclave. Conclusión: Entre los accidentes laborales estudiados, los más representativos fueron las lesiones cortofurantes y las quemaduras por autoclave. Se resalta la necesidad de actitudes que valoricen la práctica de registro de accidentes laborales, con miras a la aplicación de medidas de prevención, protección y recuperación de la salud de los profesionales involucrados en la atención directa e indirecta a la salud.

PALABRAS CLAVE: Accidentes laborales. Riesgos laborales. Enfermería.

${ }^{1}$ Enfermeira. Doutora em Enfermagem Psiquiátrica. Professora adjunta da Faculdade de Enfermagem Nossa Senhora das Graças da Universidade de Pernambuco (UPE).

E-mail: jaelquino@ig.com.br

${ }^{2}$ Enfermeira graduada pela Faculdade do Vale do Ipojuca (FAVIP). E-mail: livia_pereira_barros@hotmail.com

${ }^{3}$ Enfermeira. Especialista em Saúde Pública na Universidade de Pernambuco (UPE), Professora no Instituto Brasileiro de Tecnologia e Saúde (IBRATS).

E-mail: samara_aline06@hotmail.com

${ }^{4}$ Enfermeira. Doutoranda do Programa de Pós Graduação em Cirurgia UFPE. Professora Assistente na Universidade de Pernambuco (UPE).

Rua Marechal Deodoro, 338, Apt $^{\circ} 501^{\text {a }}$. Encruzilhada. CEP 52030-10, Recife, PE, Brasil.

Telefone: residencial (81) 3241-1854; comercial (81) 3183-3600. E-mail: emanuela.pereira@upe.br

${ }^{5}$ Enfermeira. Mestranda do Programa de Pós-graduação Associado em Enfermagem UPE/UEPB. Enfermeira Gerente Assistencial da Emergência Adulto e Pediátrica do Hospital

Prontolinda. E-mail: silviaelizabeth89@hotmail.com

${ }^{6}$ Enfermeira graduada pela Faculdade do Vale do Ipojuca (FAVIP). E-mail: betinha_enf@hotmail.com 


\section{Introdução}

Os profissionais de Enfermagem regularmente prestam assistência contínua e em condições insalubres, reforçadas por situações adversas, como elevada demanda de clientes, recursos humanos reduzidos e exigências constantes de preparo no atendimento às intercorrências, bem como pacientes de diversas complexidades. Nesse contexto, emergem os riscos de exposição e ocorrência de acidentes de trabalho ${ }^{1}$. Os riscos ocupacionais que acometem trabalhadores das instituições de saúde são oriundos de fatores físicos, químicos, biológicos e ergonômicos, os quais representam fatores capazes de prejudicar a produtividade, a qualidade da assistência prestada e a saúde ocupacional ${ }^{2}$.

Dessa forma, o ambiente da Central de Material e Esterilização (CME) é considerado como um dos setores dentro do cenário hospitalar em que as práticas específicas de processamento dos artigos ou produtos resultantes, particularmente das intervenções clínicas e cirúrgicas, aproximam e tornam os profissionais de Enfermagem mais vulneráveis aos acidentes ocupacionais ${ }^{3}$.

Em um estudo realizado em um hospital público do interior do Estado de São Paulo, evidenciou-se o elevado número de trabalhadores de Enfermagem expostos predominantemente aos acidentes no ambiente de trabalho ${ }^{4}$. Os autores ainda referem que a noção de risco pressupõe elementos isolados entre si da dinâmica global do processo de trabalho. Entretanto, os profissionais de saúde, em especial os da Enfermagem, não identificam riscos no ambiente laboral e nas atividades que executam ${ }^{2}$.

Diante do exposto, questiona-se: 'Quais são os acidentes de trabalho e os riscos ocupacionais a que estão expostos os profissionais de saúde?'. Para responder este questionamento, estabeleceu-se, como objetivo, investigar os acidentes de trabalho e os principais riscos ocupacionais a que estão expostos os profissionais de Enfermagem na CME.

\section{Método}

Trata-se de um estudo exploratório, transversal, com abordagem quantitativa, realizado em dois hospitais de referência, localizados na cidade de Caruaru, agreste pernambucano, sendo um público e um privado.

A população do estudo foi composta pela totalidade dos profissionais vinculados às Centrais de Materiais de Esterilização, compondo uma amostra de 37 profissionais de Enfermagem. Foram incluídos no estudo todos os membros da equipe de Enfermagem, de ambos os sexos, com idade acima de 18 anos, em efetivo exercício profissional nos turnos diurno e noturno, lotados, no mínimo, há então seis meses na CME e que aceitaram participar voluntariamente da pesquisa, mediante assinatura do Termo de Consentimento Livre e Esclarecido (TCLE). Foram excluídos da pesquisa os profissionais que estivessem de licenças médica, gestação e prêmio, que exercessem suas atividades no setor de forma esporádica ou que apresentassem alguma limitação durante a coleta de dados que impossibilitasse o diálogo com as pesquisadoras.

A coleta de dados foi realizada no período de agosto a de setembro de 2012, por meio de um questionário semiestruturado produzido pelas autoras. Os participantes foram abordados na CME enquanto estavam trabalhando; devido às atribuições diárias, optou-se por aplicar o instrumento nos momentos de descanso. O projeto foi aprovado pelo comitê de ética em Pesquisa da Faculdade do Vale do Ipojuca, registrado sob o CAAE $n^{\circ} 00043 / 12$, em conformidade com a Resolução ${ }^{\circ}$ 466/12 CNS/MS.

\section{Resultados}

Dos 37 profissionais de Enfermagem incluídos na pesquisa, 22 pertenciam a uma instituição pública (instituição A) e 15 a uma unidade privada (instituição B).

De acordo com os dados de identificação da amostra, apenas dois profissionais eram do sexo masculino. Com relação à faixa etária, tanto na instituição $\mathrm{A}$ quanto na $\mathrm{B}, \mathrm{o}$ intervalo etário mais prevalente foi entre $43(40,9 \%)$ e 52 $(46,7 \%)$ anos, com média de idade de 43,6 anos. Ao que se refere à situação conjugal, $63,6 \%$ dos profissionais da instituição A eram casados, enquanto $60,0 \%$ dos profissionais da B eram solteiros.

No que tange à formação profissional, foi observado que em ambas as instituições houve maior percentual de Técnicos de Enfermagem ( $86,4 \%$ na A e $66,7 \%$ na B), sendo que 4,5\% dos profissionais que trabalham na instituição A atuam como Técnico de Enfermagem, porém possuem formação superior completa.

Quanto ao tempo de atuação em unidade hospitalar, na instituição A, 45,5\% dos profissionais trabalham de 12 a 20 anos, enquanto na instituição B, 66,7\% atuam entre 4 e 10 anos. O tempo de serviço na CME em ambas as instituições apresentou uma prevalência de até 10 anos, sendo 40,9\% na instituição A e 73,3\% na B.

$\mathrm{O}$ número de vínculos empregatícios, a categoria profissional, a carga horária semanal, a unidade de lotação, bem como o turno de trabalho e a renda, são apresentados na Tabela 1.

Quanto à escala de trabalho, também observaram-se semelhanças, já que, em ambas as instituições, a prevalência de plantonistas supera os $90,0 \%$ dos profissionais estudados. Em relação à carga horária, a maior parte dos profissionais da instituição A cumpre 48 horas $(81,8 \%)$ semanais; já na $\mathrm{B}$, prevaleceu a carga horária de 36 horas $(86,7 \%)$ semanais.

Com relação à área de trabalho, observou-se que, na instituição A, 86,4\% dos profissionais trabalhavam em todas as unidades de lotação da CME, diferentemente da B, na qual os profissionais foram mais representativos em apenas uma área (40,0\%), sendo dois profissionais apenas no expurgo, dois na área de preparo e dois na área de armazenamento. 
Quanto ao turno, observou-se que, na instituição A, os profissionais trabalhavam nos dois turnos $(86,4 \%)$; já na $\mathrm{B}$, prevaleceu o diurno $(42,9 \%)$ entre os turnos dos profissionais, havendo apenas três casos em que os profissionais alegaram trabalhar nos turnos diurno e noturno.

Em relação à renda, em ambas as instituições, foi prevalente entre os profissionais a renda de um a dois salários mínimos; isso se deu pelo fato de a amostra ser composta principalmente por Auxiliares e Técnicos de Enfermagem ( $91,9 \%$ dos profissionais). Apenas os Enfermeiros indicaram renda superior a cinco salários, porém houve um Auxiliar e

Tabela 1. Distribuição dos profissionais da CME, segundo variáveis empregatícias por instituição. Caruaru-PE, 2012.

\begin{tabular}{|c|c|c|c|c|}
\hline \multirow{3}{*}{ Variáveis } & \multicolumn{4}{|c|}{ Instituição } \\
\hline & & & & \\
\hline & $\mathbf{N}$ & $\%$ & $\mathbf{N}$ & $\%$ \\
\hline
\end{tabular}

\begin{tabular}{l}
\hline Vínculos empregatícios \\
1 \\
2 \\
3 \\
Total
\end{tabular}

\section{Escala de trabalho}

Plantonista

Diarista

Total

Carga horária semanal (horas)

24

30

36

48

Total

Unidade de lotação

1 área

2 áreas

Todas as áreas

Total

Turno de trabalho

\begin{tabular}{lcrrr} 
Diurno & 3 & 13,6 & 6 & 40,0 \\
Diurno/Noturno & 19 & 86,4 & 3 & 20,0 \\
Noturno & 0 & 0,0 & 5 & 33,3 \\
Não responderam & 0 & 0,0 & 1 & 6,7 \\
Total & 22 & 100,0 & 15 & 100,0 \\
Renda (salários) & & & & \\
1 a 2 & 16 & 72,8 & 10 & 66,6 \\
3 a 4 & 5 & 22,7 & 4 & 26,7 \\
5 ou + & 1 & 4,5 & 1 & 6,7 \\
Total & 22 & 100,0 & 15 & 100,0 \\
\hline
\end{tabular}

sete Técnicos que afirmaram ganhar de três a quatro salários mínimos.

A Tabela 2 apresenta a adesão ao uso de Equipamentos de Proteção Individual (EPIs) pela quantidade de equipamentos utilizados e pelos acidentes de trabalhos, bem como o tipo de acidente, o turno em que ocorreu e a realização da notificação desses acidentes.

Todos os profissionais alegaram fazer uso dos EPIs, em ambas as instituições, e observou-se prevalência do uso de cinco ou mais destes (90,9\% na instituição A e 53,3\% na B). Os EPIs mais utilizados foram as luvas, as máscaras, os óculos e os propés indicados por quase todos os profissionais.

$\mathrm{O}$ número de pessoas que sofreu acidentes em ambas as instituições merece destaque. Na instituição A, 27,3\% dos sujeitos afirmaram ter sofrido algum tipo de acidente,

Tabela 2. Distribuição dos profissionais da CME, segundo adesão de EPI, acidentes de trabalho e fatores associados. Caruaru-PE, 2012.

\begin{tabular}{|c|c|c|c|c|}
\hline \multirow{3}{*}{ Variáveis } & \multicolumn{4}{|c|}{ Instituição } \\
\hline & \multicolumn{2}{|c|}{$\mathbf{A}$} & \multicolumn{2}{|c|}{ B } \\
\hline & $\mathbf{N}$ & $\%$ & $\mathbf{N}$ & $\%$ \\
\hline \multicolumn{5}{|l|}{ Adesão de EPIs } \\
\hline Sim & 22 & 100,0 & 15 & 100,0 \\
\hline Não & 0 & 0,0 & 0 & 0,0 \\
\hline Total & 22 & 100,0 & 15 & 100,0 \\
\hline \multicolumn{5}{|l|}{ Acidentes de trabalho } \\
\hline Sim & 6 & 27,3 & 7 & 46,7 \\
\hline Não & 16 & 72,7 & 8 & 53,3 \\
\hline Total & 22 & 100,0 & 15 & 100,0 \\
\hline \multicolumn{5}{|l|}{ Tipos de acidente } \\
\hline Choque com material & 0 & 0,0 & 1 & 14,3 \\
\hline Lesão por perfurocortante & 5 & 83,3 & 2 & 28,6 \\
\hline Queimadura na autoclave & 1 & 16,7 & 4 & 57,1 \\
\hline Total & 6 & 100,0 & 7 & 100,0 \\
\hline \multicolumn{5}{|l|}{ Turno do acidente } \\
\hline Diurno & 3 & 50,0 & 5 & 71,4 \\
\hline Noturno & 3 & 50,0 & 2 & 28,6 \\
\hline Total & 6 & 100,0 & 7 & 100,0 \\
\hline \multicolumn{5}{|l|}{ Notificação } \\
\hline Sim & 5 & 83,3 & 4 & 57,1 \\
\hline Não & 1 & 16,7 & 3 & 42,9 \\
\hline Total & 6 & 100,0 & 7 & 100,0 \\
\hline \multicolumn{5}{|l|}{ Processo de notificação realizado } \\
\hline $\mathrm{CCIH}$ & 1 & 20,0 & 0 & 0,0 \\
\hline Enfermeiro de plantão & 3 & 60,0 & 0 & 0,0 \\
\hline CCIH e Enfermeiro de plantão & 1 & 20,0 & 0 & 0,0 \\
\hline $\begin{array}{l}\text { Segurança do trabalho e Enfermeiro } \\
\text { de plantão }\end{array}$ & 0 & 0,0 & 4 & 100,0 \\
\hline Total & 5 & 100,0 & 4 & 100,0 \\
\hline
\end{tabular}


enquanto na B foram relatados $46,7 \%$, sendo $83,3 \%$ com perfurocortantes na $\mathrm{A}$ e $57,1 \%$ por queimaduras em autoclave na B.

$\mathrm{Na}$ instituição $\mathrm{A}$, não houve diferença percentual na ocorrência dos acidentes segundo o turno de trabalho; porém, na instituição $\mathrm{B}$, a maior parte dos acidentes ocorreu no plantão diurno $(71,4 \%)$.

Com relação às notificações das lesões, 83,3\% foram notificadas na instituição $\mathrm{A}$, enquanto $57,1 \%$ foram registradas na $\mathrm{B}$. Na instituição $\mathrm{A}, 60 \%$ dos acidentes foram notificados pelo Enfermeiro de plantão, enquanto que na B, $100,0 \%$ foram notificados pela segurança do trabalho.

A Figura 1 apresenta a distribuição dos riscos percebidos pelos profissionais na CME. Observa-se que os riscos mais percebidos pelos profissionais foram o risco de incêndio (91,9\%); o risco de contato com substâncias químicas e exposição a ruídos (ambos com 89,2\%); o esforço físico levando à fadiga; a lesão por perfurocortante, e a queda de materiais (todos percebidos por $86,5 \%$ dos entrevistados).

A agressividade, o assédio sexual ou a violência no trabalho só foram relatados por $5,4 \%$ dos profissionais.

A distribuição dos danos à saúde "provocados" pelo trabalho na CME está apresentada na Figura 2. Verifica-se que a fadiga muscular (43,2\% dos profissionais), as varizes $(37,8 \%)$ e o estresse $(32,4 \%)$ foram os danos à saude que os profissionais mais relataram serem "provocados" pelo trabalho na CME. Doenças do coração, do fígado, das vias respiratórias, do sistema nervoso e da pele, e intoxicações por metais pesados foram relatadas por $5,4 \%$ dos profissionais. Os problemas menos citados foram agressões e doenças infecciosa (ambas com 2,7\%).

A Figura 3 apresenta a distribuição dos danos à saúde “agravados" pelo trabalho na CME. Os danos à saúde mais citados pelos profissionais da CME foram as varizes (16,2\%), os problemas oculares $(13,5 \%)$ e as lombalgias $(10,8)$. Os transtornos do sono e as lesões na coluna vertebral foram relatados por $8,1 \%$ dos entrevistados. Os demais problemas foram pouco citados.

\section{Discussão}

No que se refere ao predomínio de profissionais de Enfermagem do sexo feminino, a presente pesquisa corrobora com estudo realizado no interior do Estado de São Paulo, que constatou a prevalência de $91,5 \%$ de mulheres ao analisar os profissionais da CME no ano de $2007^{5}$.

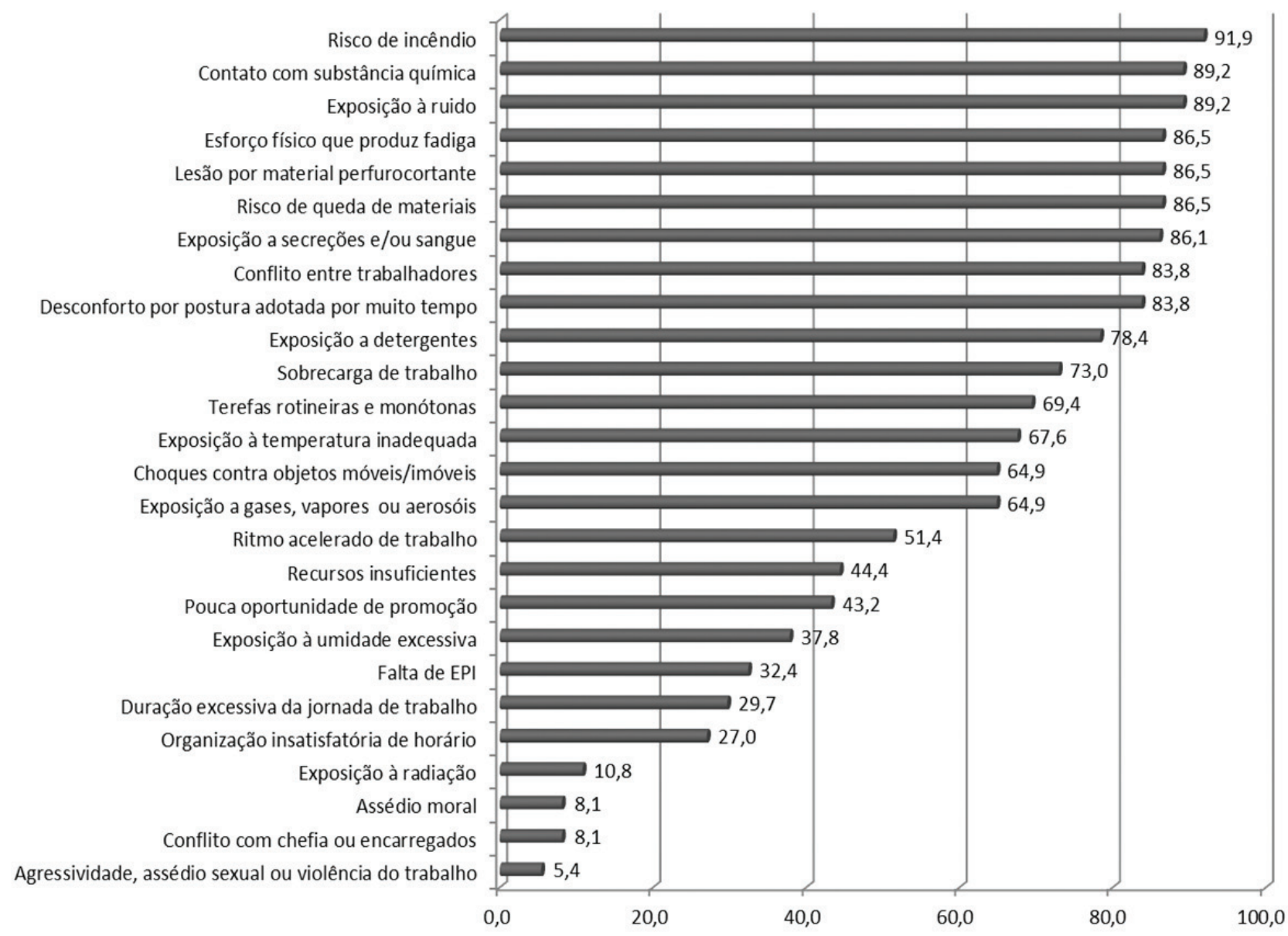

Figura 1. Distribuição dos riscos percebidos pelos profissionais na CME. Caruaru-PE, 2012. *Excluídas as casas zeradas. 


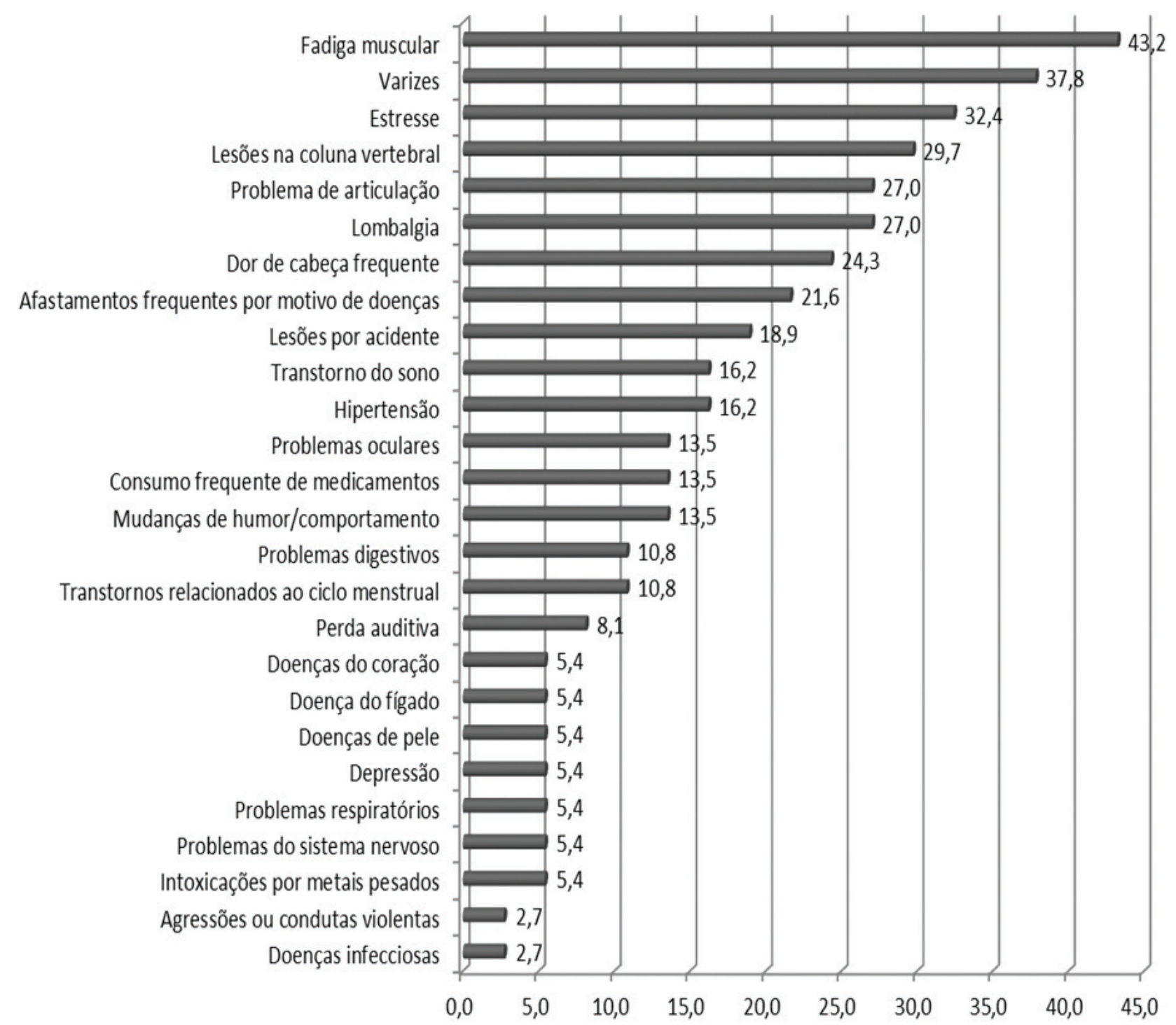

Figura 2. Distribuição dos danos à saúde "provocados" pelo trabalho na CME. Caruaru-PE, 2012. *Excluídas as casas zeradas.

A população do presente estudo apresentou uma idade média de 43,6 anos, o que, por sua vez, merece destaque das unidades contratantes, uma vez que, após os 35 anos de idade, as funções cardiovasculares, pulmonares, musculares, bem como as adaptações físicas, estão diminuídas ${ }^{6}$.

O número de vínculos empregatícios estabelecidos pelos participantes esteve em contraponto com a realidade brasileira, uma vez que, neste estudo, prevaleceu a vinculação com apenas um participante. Uma pesquisa realizada com trabalhadores do hospital universitário de São Paulo descreve que a mulher, de maneira geral, insere-se no mercado de trabalho como forma de contribuir para o aumento da renda familiar, submetendo-se a dupla ou tripla jornada de trabalho, o que propicia desgaste físico e emocional, expondo-a a maiores riscos de acidentes ${ }^{7}$.

O exercício profissional na CME está organizado em unidades de lotação específicas, que compõem o cenário físico do serviço em questão. Pesquisadores fundamentam que a área física do CME deve permitir o fluxo contínuo e unidirecional do artigo; para isto, é necessário que haja barreiras físicas entre as áreas: suja (expurgo), limpa (preparo, dobradura e esterilização) e sistema de guarda, do qual se exige maior rigor, devido à manutenção da esterilidade dos artigos ${ }^{8}$.

No que se refere ao uso de Equipamentos de Proteção Individual (EPIs), os mesmos protegem e minimizam os acidentes de trabalho entre os profissionais de Enfermagem. Pesquisadores sugerem a hipótese de que trabalhadores com mais tempo de serviço e experiência possam se sentir mais seguros e, de certa forma, negligenciar certas precauções por confiar demasiadamente em sua destreza, acidentando-se algumas vezes ${ }^{9,10}$.

Em relação ao predomínio de acidentes de trabalho e a associação com o turno de trabalho, observou-se que a maior 


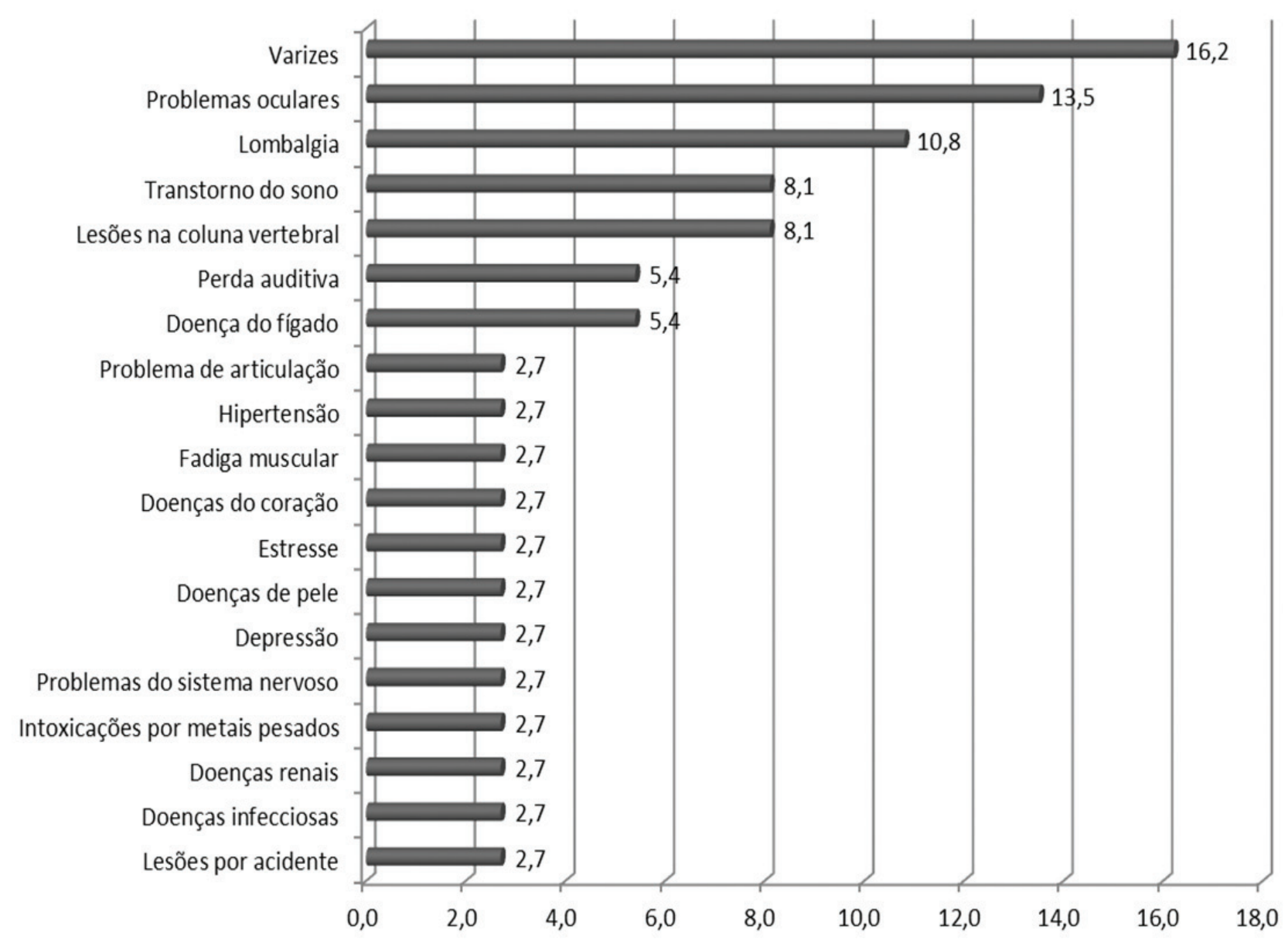

Figura 3. Distribuição dos danos à saúde “agravados” pelo trabalho na CME. Caruaru-PE, 2012. *Excluídas as casas zeradas.

parte dos acidentes de trabalho, na instituição B, aconteceu durante plantões diurnos. Segundo Araújo ${ }^{11}$, existe uma relação enfatizada entre os turnos em que acontecem os acidentes de trabalho, porque há possibilidades maiores de os acidentes ocorrerem no período da manhã devido à demanda de atividades.

De acordo com o processo de notificação dos acidentes de trabalho, um estudo ${ }^{12}$ verificou que os motivos que levaram à não notificação estavam relacionados a crenças, falta de conhecimentos e informações sobre o risco de contaminação e forma de registro dos dados, o que corrobora com os resultados obtidos nesta pesquisa, em que $42,9 \%$ dos casos, na instituição $\mathrm{B}$, não foram registrados.

A percepção dos riscos pelos profissionais da $\mathrm{CME}$ é fundamental, pois reforça o entendimento de que tais colaboradores reconhecem o ambiente de trabalho em que se encontram e as potenciais ameaças ${ }^{13}$. Os participantes deste estudo destacaram como principais riscos a que estão expostos a possibilidade de incêndio e explosão (91,9\%), os riscos químicos e físicos (ambos com 89,2\%). Tais relatos, por sua vez, corroboram com um estudo realizado em São Paulo, no hospital universitário ${ }^{13}$.
As atividades decorrentes do trabalho são, às vezes, responsáveis por danos físicos, em virtude da falta de conhecimento sobre medidas preventivas e do uso incorreto de equipamentos de proteção ${ }^{14,15}$; estes, quando selecionados e usados segundo as recomendações, minimizam os riscos ocupacionais e contribuem para uma assistência de qualidade.

Os danos à saúde, como fadiga muscular, varizes, estresse e lesões na coluna vertebral, foram as doenças mais citadas, uma vez que as mesmas são resultantes das exposições aos agentes físicos, ergonômicos, químicos e biológicos presentes na ambiência do trabalho ${ }^{16}$.

Entre os danos à saúde "provocados" pelo trabalho na CME, as doenças ocupacionais ocupam destaque e estão relacionadas com a frequência significativamente elevada nas atividades que exigem grande esforço físico, em ritmo ou intensidades excessivas, e em condições claramente não ergonômicas. Assim, as condições de trabalho apresentadas, como longos períodos em uma determinada atividade em pé, a climatização artificial e o esforço físico, demonstram uma tendência de comprometimento da saúde do trabalhador de Enfermagem ${ }^{17}$.

Estes achados evidenciam a complexidade dos fatores que têm atuado de maneira sinérgica no surgimento de 
doenças ocupacionais, enfatizando as situações de risco a que estão expostos os profissionais de Enfermagem nas $\mathrm{CMEs}^{16}$. Diante desta perspectiva, é possível inferir que vivenciamos uma realidade contraditória na saúde, pois, teoricamente, fundamentamo-nos na promoção, na proteção e na recuperação da saúde; no entanto, a prática encontra-se desarticulada, ergonomicamente inapropriada e adoecedora.

\section{Conclusão}

A presente pesquisa contribuiu, de maneira efetiva, no processo de reconhecimento dos riscos ocupacionais aos quais se encontram expostos os trabalhadores de Enfermagem. Dessa forma, é importante estruturar e oferecer treinamento eficaz, bem como avaliar o impacto destes danos junto aos trabalhadores, considerando-se os problemas levantados e a possibilidade de intervenção nos mesmos.

Dentre os acidentes de trabalho levantados, as lesões com perfurocortantes e as queimaduras por autoclave foram as mais representativas nas instituições. Os profissionais de Enfermagem foram bastante críticos no levantamento de riscos ocupacionais a que estão expostos, dando ênfase aos riscos de incêndio, contato com substâncias químicas e biológicas, exposição a ruídos, esforço físico e lesões com perfurocortantes, além do risco de queda dos materiais, do desconforto por postura adotada e da sobrecarga de trabalho. Estes fatores parecem ter contribuído para o aparecimento de danos à saúde, como varizes, problemas oculares, lombalgia, transtorno do sono e lesões na coluna vertebral, dentre outros.

Sugere-se, dessa forma, a ampliação de novas pesquisas, para proporcionar o aprimoramento e a funcionalidade na gestão de riscos ocupacionais, assim como a sua aplicabilidade em outras categorias profissionais. Reforçase também a necessidade da realização de estudos sobre o conhecimento dos profissionais acerca da utilização dos EPIs, visto que os trabalhadores os utilizam de forma parcial ou inapropriada.

No que se refere às notificações, percebe-se a necessidade de atitudes que valorizem a prática do registro dos acidentes laborais, visando à implantação de medidas de prevenção, proteção e recuperação da saúde dos profissionais envolvidos na assistência direta e indireta à saúde. Sabe-se que o funcionamento adequado da Central de Materiais e Esterilização é de fundamental importância no planejamento dos diversos setores hospitalares. Desse modo, valorizar a qualidade de vida dos profissionais, bem como fortalecer os vínculos destes com a educação permanente e continuada, é imperativo na garantia da saúde dos envolvidos.

\section{Referências}

1. Lima FA, Pinheiro PNC, Vieira NFC. Acidentes com material perfurocortante: conhecendo os sentimentos e as emoções dos profissionais de enfermagem. Esc Anna Nery R Enferm. 2007;11(2):205-11. http://dx.doi.org/10.1590/ S1414-81452007000200004
2. Chiodi MB, Marziale MHP. Riscos ocupacionais para trabalhadores de Unidades Básicas de Saúde: revisão bibliográfica. Acta Paul Enferm. 2006;19(2):212-7. http://dx.doi.org/10.1590/ S0103-21002006000200014

3. Bartolomei SRT, Lacerda RA. O enfermeiro da Central de Material e Esterilização e a percepção do seu papel social. Rev. Gaúcha Enferm. 2006;27(2):258-65. PMid:17025043.

4. Sarquis LMM, Felli VEA. Acidentes de trabalho com instrumentos perfurocortantes entre os trabalhadores de enfermagem. Rev. Esc. Enfermagem USP. 2002;36(3):222-30. PMid:12754915. http:// dx.doi.org/10.1590/S0080-62342002000300003

5. Talhaferro B, Barboza DB, Oliveira AR. Adesão ao uso dos equipamentos de proteçãoindividual pela enfermagem. Rev. Ciênc. Méd. 2008;17(3-6):157-66.

6. Santos KL, Soler Z. Absenteísmo na enfermagem: enfoque nas causas de ordem psicológica. Rev. Enferm. Brasil. 2003;6(2):336-43.

7. Balsamo AC, Felli VEA. Estudo sobre os acidentes de trabalho com exposição aos líquidos corporais humanos em trabalhadores da saúde de um hospital universitário. Rev Latinoam Enfermagem. 2006;14(3):346-53. http://dx.doi.org/10.1590/ S0104-11692006000300007

8. Sociedade Brasileira de Enfermeiros de Centro Cirúrgico, Recuperação Anestésica e Centro de Material e Esterilização - SOBECC. Práticas Recomendadas da SOBECC: centro cirúrgico, recuperação anestésica e centro de material e esterilização. São Paulo: SOBECC; 2007.

9. Zapparoli AS. Promoção da saúde do trabalhador de enfermagem: análise da prática segura do uso de luvas na punção venosa periférica [dissertação]. Ribeirão Preto: Escola de Enfermagem de Ribeirão Preto, Universidade de São Paulo; 2005.

10. Araújo AA, Santos IBC, Oliveira EF. Reflexões sobre o desempenho dos colaboradores no Centro de Material e Esterilização. Rev SOBECC. 2006;11(4):31-6.

11. Ribeiro EJG, Shimizu HE. Acidentesde trabalho com trabalhadores de enfermagem. Revista Bras Enferm. 2007;60(5):535-40. PMid:18041553. http://dx.doi.org/10.1590/ S0034-71672007000500010

12. Marziale MHP. Subnotificação de acidentes com perfurocortantes na enfermagem. Rev Bras Enferm. 2003;56(2):164-8. PMid:14692282. http://dx.doi.org/10.1590/S0034-71672003000200011

13. Nishide VM, Benatti MCC.Riscos ocupacionais entretrabalhadores de enfermagem de uma unidade de terapia intensiva. Rev Esc Enferm USP. 2004;38(4):406-14. http://dx.doi.org/10.1590/ S0080-62342004000400006

14. Almeida CB, Pagliuca LMF, Leite ALAS. Acidentes de trabalho envolvendo os olhos: avaliação de riscos ocupacionais com trabalhadores de enfermagem. Rev. Latino-Am Enfermagem. 2005;13(5):708-16. http://dx.doi.org/10.1590/ S0104-11692005000500015

15. Oliveira BRG, Murofuse NT. Acidentes de trabalho e doença ocupacional: estudo sobre o conhecimento do trabalhador hospitalar dos riscos à saúde de seu trabalho. Rev Latino-Am Enfermagem. 2001;9(1):109-15. http://dx.doi.org/10.1590/ S0104-11692001000100016

16. Tipple AFV, Aguliar HT, Souza ACS, Pereira MS, Mendonça ACC, Silveira C. Equipamentos de proteção em centros de material eEsterilização: disponibilidade, uso e fatores intervenientes à adesão. Cien Cuid Saude. 2007;6(4):441-8.

17. Souza FMS. Condições de trabalho de ambiente cirúrgico e a saúde dos trabalhadores de enfermagem [dissertação]. Rio de Janeiro: Universidade Estadual do Rio de Janeiro; 2011. 